\title{
Preparing Students for the Complexity of the Systems Medicine Field through a Research Experience For Undergraduates (REU)
}

\section{Mr. Nolan Alexander LaSota, University of Pittsburgh}

Nolan LaSota is currently a student at the University of Pittsburgh. He plans on majoring in chemical engineering.

Prof. Robert S. Parker, University of Pittsburgh

Dr. Cheryl A Bodnar, University of Pittsburgh

Cheryl A. Bodnar, PhD, CTDP is an Assistant Professor (Teaching Track) in the Department of Chemical and Petroleum Engineering at the Swanson School of Engineering at the University of Pittsburgh. She obtained her certification as a Training and Development Professional (CTDP) from the Canadian Society for Training and Development (CSTD) in 2010, providing her with a solid background in instructional design, facilitation and evaluation.

Dr. Bodnar's research interests relate to the incorporation of active learning techniques in undergraduate classes (problem based learning, games and simulations, etc.) as well as integration of innovation and entrepreneurship into the Chemical and Petroleum Engineering as well as broader engineering curriculum. In addition, she is actively engaged in the development of a variety of informal science education approaches with the goal of exciting and teaching K-12 students about regenerative medicine and its potential. 


\title{
Preparing Students for the Complexity of the Systems Medicine Field through a Research Experience For Undergraduates (REU)
}

\begin{abstract}
Over the next few decades, the total cost of healthcare is anticipated to increase dramatically. ${ }^{1}$ Increasing the efficiency and effectiveness of healthcare delivery, via model-based systems engineering methods, has the potential to slow this increase. One method to accomplish this is through the employment of engineers and scientists that have an understanding of mathematical modeling, biology, and clinical practice who can interact with caregivers and administrators to improve clinical outcomes. This field, also known as "systems medicine", is an area that is currently underserved within the undergraduate education community.
\end{abstract}

In response to the need for engineers and scientists cross-trained to effectively work on the medical interface, a National Science Foundation funded Research Experience for Undergraduates (REU) program (NSF EEC-1156899) has been run for the last three years (20122014) at the University of Pittsburgh. This program consisted of students being paired with one or more mentor(s) on a specific technical project over a ten-week period while receiving professional development opportunities through a weekly workshop series. The professional development opportunities included skills that are relevant to engineering or scientific careers such as scientific writing and presentation skills, as well as opportunities that provided students with the background and scope of the diverse systems medicine field. Students presented their work in a local undergraduate research poster session and provided a final presentation on their research results. Select students also presented their research at national meetings.

To determine the effectiveness of the REU program for preparing students for the complexity of the systems medicine field, students completed concept maps detailing their vision of the systems medicine field at the start and end of their ten-week research experience. Completion of the concept map was done subsequent to a training exercise that was performed to familiarize students with concept maps and how they are constructed. Utilizing the Integrated Rubric for Scoring Concept Maps ${ }^{2}$, a three-point scale was used to score the concept maps on the basis of comprehensiveness, organization and correctness. Each concept map was scored by two individuals and an inter-rater reliability between fair and good reliability was found for all dimensions. The concept map scoring results for the three years of the REU program showed a slight but not statistically significant increase in the overall concept map scores from a mean of 6.13 at the start of the program to 6.32 upon completion of the program based on both the t-test and non-parametric Wilcoxon-Rank Sum test. Analysis of individual dimensions of the concept maps revealed that comprehensiveness scores increased from a mean of 1.68 to 1.92 ( $\mathrm{p}=0.08$ based on Wilcoxon-Rank Sum test), while organization and correctness scores did not demonstrate a significant change, if any, remaining at means of approximately 2.00 and 2.40, respectively. Based on these results, the REU program broadened the perspective that undergraduate researchers have of the systems medicine field, although the result is not statistically significant at present, likely due to the small sample size $(\mathrm{N}=60)$. 


\section{Introduction}

Engineers often address multi-disciplinary open-ended design problems throughout their careers. The National Academy of Engineering Grand Challenges website characterizes some of the toughest problems facing engineers, including reverse engineering the brain and engineering better medicines. Attacking these problems requires multifaceted knowledge of engineering principles as well as basic science principles from multiple fields. ${ }^{3}$ Similarly medical challenges in discovery and translation can benefit from close collaboration between engineers, basic scientists, and clinicians.

According to the Coordinating Action Systems Medicine of Europe, systems medicine is defined as, "the implementation of Systems Biology approaches in medical concepts, research and practice. This involves feedback between clinical investigations and practice with computational, statistical and mathematical analysis and modeling." "The generalized clinical issues that can be addressed in the systems medicine field are diverse, including disease progression and remission, disease spread and cure, treatment responses, and disease prevention. ${ }^{4}$ However, medical practice is for a specific disease impacting an individual patient. As a result, it is crucial to have a thorough understanding of disease-specific characteristics (e.g., what agents can be used to treat lung cancer and what are the trade-offs of using them) in order to have clinical impact at the patient scale. Similarly, the discovery of early warning signs for diseases, such as diabetes, depression and cancer, can lead to more effective prevention and protection strategies. ${ }^{5}$ One of the major diseases where systems medicine has had an impact is cancer. Recent developments in technology allow rapid screening of potential drug candidates that kill cancer cells. ${ }^{6}$ Modeling of the drug activity and cellular response provides insight for engineers and clinicians on the action, and interaction, of cancer treatments in vitro. ${ }^{6}$ Though it has been shown to be difficult to accomplish, scaling this knowledge to the in vivo scenario of patient treatment provides an opportunity to use knowledge of drug administration and action to change clinical treatment schedules for agents in an effort to reduce toxicity while maintaining antitumor efficacy. $7,8,9$

To be able to continually develop personnel to work within this critical area, it is necessary to provide students with the opportunity to gain exposure of this field at an undergraduate level. In an effort to meet this need, the University of Pittsburgh implemented a Research Experience for Undergraduates (REU) program through funding received from the National Science Foundation with a specific focus on systems medicine. Each year from 2012-2014, a group of students went through a ten week mentored program of research-based learning. The program combined independent research with a faculty mentor, along with professional development training in modeling and analysis, teamwork, and communication skills amongst others that are beneficial to becoming a successful engineer.

To determine the effectiveness of the REU program, students were asked to complete concept maps for the field at the start and end of their ten week research experience. Concept mapping is, "a graphical organizer for thoughts, theories, and/or concepts." 2 The point of the concept map is to observe students' understanding of the subject matter area and how they feel the concepts within this field connect to one another. Upon completion of the REU program in 2014, the maps were then scored on three different criteria: comprehensiveness, organization, and correctness 
according to the concept map rubric that was developed by Besterfield-Sacre et al. ${ }^{2}$ In this rubric, comprehensiveness is defined as "broadness of knowledge and the completeness of the subject definition." Organization consists of concept integration and how well the thoughts were connected to one another. Lastly, correctness is how accurate the information contained within the map was. ${ }^{2}$

Concept map scores from before and after the REU program were then compared to determine if any changes occurred as a result of students' participation within this program. REU programs at other institutions have shown to be able to measure change over this short time period. Based on a study at Missouri University of Science and Technology where a REU program was run from 2011-2013, their results showed improvement in the students' knowledge of the additive manufacturing processes. In the 2012-2014 study, the Missouri University program evaluated students with a survey before and after the program, along with weekly presentation evaluations. The Missouri study obtained qualitative data through interviews with the participants prior to and after completion of the REU program. It was also stated that the program seemed to improve year after year and succeeded in increasing the students' awareness of additive manufacturing. ${ }^{10}$

\section{Research Questions}

The research questions that we had in this study were:

1. Does the REU program contribute to increasing students' understanding/perception of the systems medicine field through an increase in concept map scores?

2. Was there any specific year of implementation that was shown to be more effective than the others in developing students' understanding/perception of the systems medicine field?

\section{Methods}

\section{Study Design}

This study was conducted with three cohorts of students that participated in an REU program focused on the systems medicine field. Each year, a cohort of nine to eleven students, participated in independent research and had the opportunity to learn more about the systems medicine field through integrated professional development workshops and seminars. As part of the REU program, students were asked to create concept maps using the central topic of systems medicine. Before their first mapping session, students were led through an interactive example of constructing a concept map using a well-known item, french fries, as the central idea; this provided students an opportunity to observe the synthesis of a concept map and how the concepts are integrated with one another providing them with an understanding of what was expected. Each of the participants made one map at the start of the program on either the first or second day after arrival and upon completion of the program during the last week of the REU experience.

All concept maps were labeled with identifiers that would enable analysis to be performed on a blinded basis and human subjects' approval was obtained prior to commencing this study. These 
concept maps were then evaluated using the Concept Map rubric for comprehensiveness, organization and correctness. ${ }^{2}$

The purpose of the Concept Map rubric is to accurately judge each map on "knowledge integration." Before concept maps, students' understanding of concepts was evaluated using standardized testing, which is limited and does not always match what is taught by the instructor. Other methods include behavioral observations and portfolios that track student's progress in an area of study. However, concept mapping seems to be the best method of testing knowledge integration because it organizes all of one's thoughts and ideas into an interconnected web of hierarchies that shows integration between different areas of the central topic or field. The two ways of scoring concept maps are a traditional counting method, which counts the number of concepts, interconnections, and levels of branches, and a holistic approach, which focuses on the overall completeness, breadth, and quality of the map. The current rubric for scoring concept maps combines elements of both the traditional counting and holistic approaches to form the best evaluation.

\section{Concept Map Rubric and Scoring}

We found that most maps analyzed didn't fit uniquely into any of the three scores provided within the rubric $(1,2$, or 3$)$. To capture these intermediate map results, half-point scores (1.5 and 2.5) were used when maps contained aspects of both the upper and lower integer levels but didn't fit completely into either category. This change was made prior to any scoring being performed, which allowed for all maps to be consistently evaluated.

To determine the impact the REU program had on students' understanding/perception of the systems medicine field, total concept map scores as well as individual dimension scores for each concept map were compared from before the program and after completion of the program using a paired-samples t-test and the non-parametric Wilcoxon Rank Sum test for related samples due to the small sample size. Overall performance across all years of the REU program was performed alongside analysis of results for each REU cohort.

\section{Reliability Analysis}

Each concept map was scored by two individual coders. One coder was an undergraduate student researcher and the other coder was a chemical engineering faculty member that had peripheral involvement in the REU program. When the coders disagreed on a score for a dimension of the concept map, they would meet with the mediator, the principal investigator for the REU program, to discuss the results and reach an agreement. When final agreement was reached this score was recorded as the final score for that dimension of the map.

The inter-rater reliability scores for this analysis are shown in Table 1 based on the intraclass correlation coefficient which is often used to measure consistency in scores. All ICC values were calculated using the "average measure" function in SPSS. ${ }^{11}$ 
Table 1. Inter-Reliability Values for Concept Map Scoring

\begin{tabular}{|l|c|}
\hline Rubric Dimension & $\begin{array}{c}\text { Reliability Score Based on Intraclass } \\
\text { Correlation Coefficient (ICC) }\end{array}$ \\
\hline Comprehensiveness & .747 \\
\hline Organization & .691 \\
\hline Correctness & .543 \\
\hline Overall & .732 \\
\hline
\end{tabular}

The results from this analysis demonstrate that there is fair to good reliability between the two coders that performed this analysis as Fleiss documented that ICC values between 0.4 and 0.75 can be attributed to being in the fair to good reliability range. ${ }^{12}$ The lowest reliability score found was 0.543 and this corresponded to the level of correctness that was observed in the concept maps. As one coder was an undergraduate student researcher with no previous experience in the systems medicine field and the other coder was a chemical engineering faculty member who had been helping with the REU program it is possible that this difference in experience level and understanding of the field may have contributed to the results obtained.

\section{Results and Discussion}

To establish whether the REU program contributed to increasing students' understanding / perception of the systems medicine field, the concept map scores of all students that participated in the REU program from 2012 to 2014 were analyzed. The concept map results, as shown in Table 2, are broken down on the basis of comprehensiveness, organization, correctness and total score, where total score represented the sum of all of the individual dimension scores.

Table 2. Concept Map Scoring Rubric Results for Overall REU Program

\begin{tabular}{|c|l|c|c|c|c|}
\hline Dimension & \multicolumn{1}{|c|}{ Definition } & $\begin{array}{c}\text { Pre-REU } \\
\text { Score }\end{array}$ & $\begin{array}{c}\text { Post-REU } \\
\text { Score }\end{array}$ & $\begin{array}{c}\text { p-value } \\
\text { (t) }\end{array}$ & $\begin{array}{c}\text { p-value } \\
\text { (Wilcoxon) }\end{array}$ \\
\hline Comprehensiveness & $\begin{array}{l}\text { Broadness of } \\
\text { knowledge; } \\
\text { completeness of } \\
\text { subject definition }\end{array}$ & 1.68 & 1.92 & .065 & .080 \\
\hline Organization & $\begin{array}{l}\text { Branches are well } \\
\text { defined and properly } \\
\text { placed }\end{array}$ & 2.05 & 2.00 & .732 & .572 \\
\hline Correctness & $\begin{array}{l}\text { Accuracy of } \\
\text { information; spelling } \\
\text { and/or grammar } \\
\text { mistakes }\end{array}$ & 2.40 & 2.40 & 1.000 & .985 \\
\hline Total & $\begin{array}{l}\text { Sum of the concept } \\
\text { map scores across all } \\
\text { dimensions analyzed }\end{array}$ & 6.13 & 6.32 & 0.452 & 0.581 \\
\hline
\end{tabular}


The total score from the pre to the post concept map showed a slight, but not significant, increase changing from a mean value of 6.13 to 6.32 after completion of the program. This difference can be attributed to the change observed in the comprehensiveness dimension where the mean score increased from 1.68 to 1.92 . The change in comprehensiveness could be attributed to the combined effect of at least two program factors: (i) the mentored research experience increasing the depth of knowledge of REU participants in their particular research area; and (ii) the workshop series that provided some sessions on different aspects of the systems medicine field from the perspective of both engineers and medical doctors, thereby broadening the scope of students' knowledge breadth.

Results obtained for the other two dimensions of the rubric showed no noticeable changes between the start and the end of the program. Organization, which examines connection and integration between branches, may not have changed as the students may not have been able to make any further connections between the new material that they were exposed to and their existing perception of systems medicine. One thing to consider for subsequent REU programs would be increase the focus on integration and connectivity of systems medicine topics beyond the current design to expand participants' knowledge.

The correctness dimension deals with the question, "Are the things that the students' know about systems medicine correct?" The high mean score obtained on this dimension of 2.40 out of a possible 3.00 points seems reasonable considering the context of the evaluation. In some cases, maps where the student scored fairly low on both the organization and comprehensiveness sections would score high on correctness because all of the ideas were accurate. Examples of a pre and post-concept map are shown in Figures $1 \mathrm{a}$ and $\mathrm{b}$. 


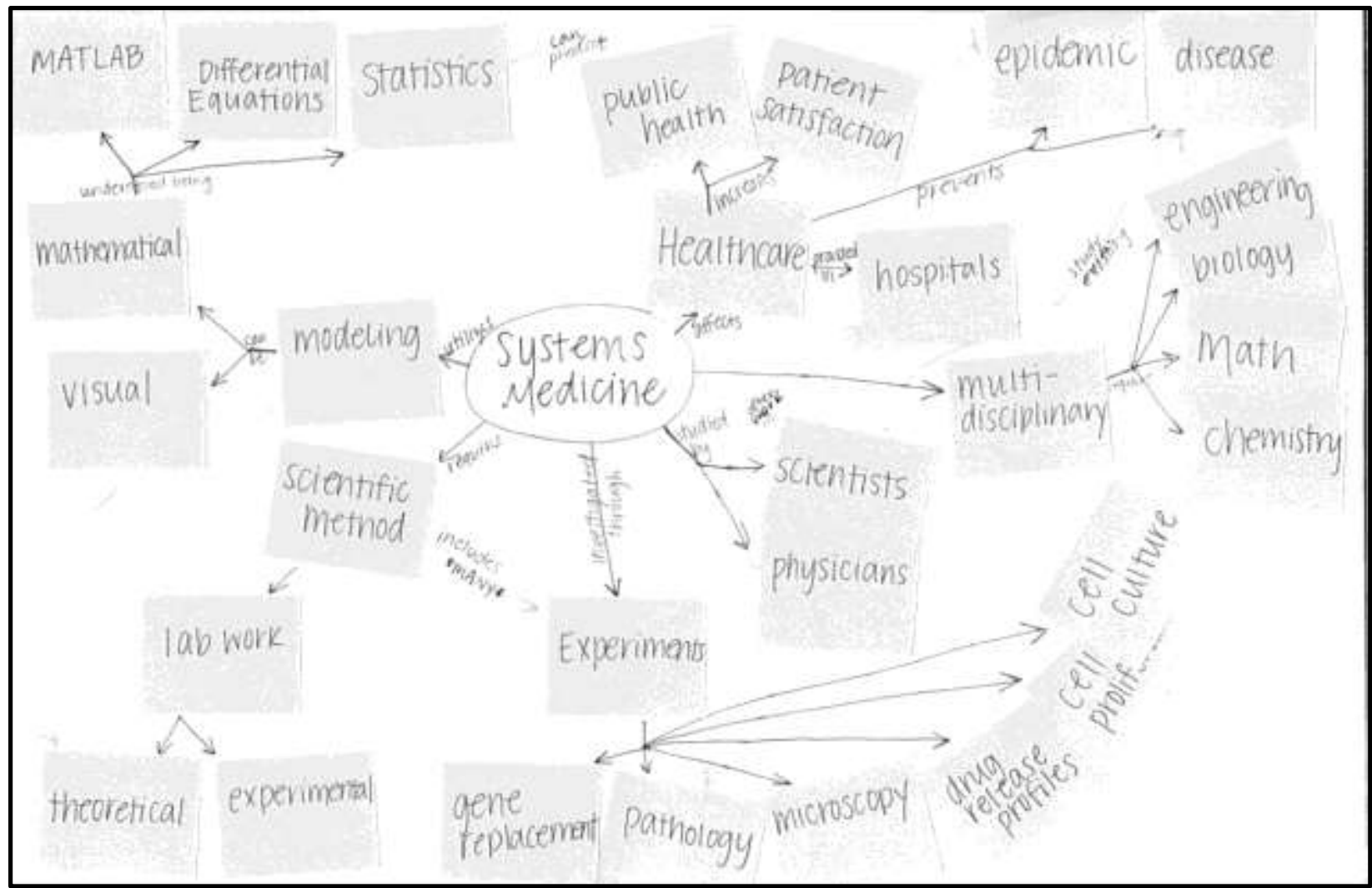

Figure 1a. Example of a Systems Medicine Concept Map at Start of Program.

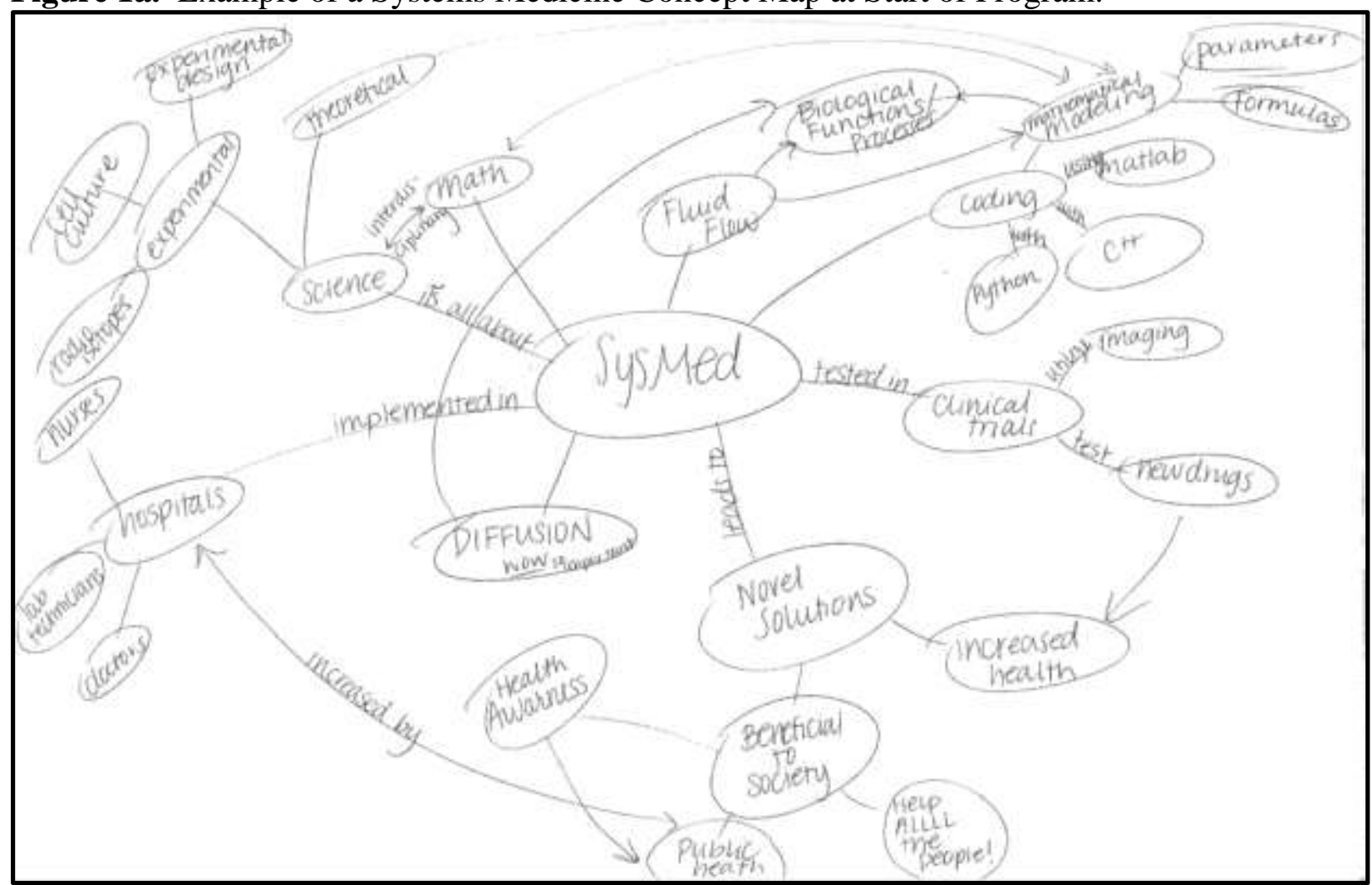

Figure 1b. Example of a Systems Medicine Concept Map at End of Program. 
Figure 1a shows a map that is primarily "linear" in terms of the branches out from the centerpoint of Systems Medicine. There are some linkages between the branches, but many are missing, such as a complete disconnect between experiments (bottom center) and other branches containing lab work, analysis tools, coursework, etc. The connectivity of Figure $1 \mathrm{~b}$, a postprogram map, is far superior, with crosslinks between most every branch of the diagram. Given the multidisciplinary nature of the Systems Medicine field and program, recognition of these links, and the knowledge that there are many places and people from which expertise can be gained, is important as one addresses problems in the field. It should be noted that neither the pre- nor the post-program maps are perfect, however, in that there are items missing from both maps (notably the role of industry in the development/deployment of treatment solutions). If map analysis consistently finds the same items missing from a cohort, this topic is a candidate for increased workshop focus in following years.

Analysis of the concept map scoring was also performed on a yearly basis to determine if a particular cohort of students showed more advancement in their understanding of the systems medicine field in comparison to other cohorts that participated in this program. Table 3 provides the results from this analysis broken down by year and dimension of the rubric. All of the years showed slight, but not significant, increases in total concept map scores except for 2013 where the mean total score changed from 6.32 at the start of the program to 5.86 at the end of the program. These changes are primarily driven by decreases in both organization and correctness for the 2013 cohort. During the 2013 program, the principal investigator did miss just over two weeks of the program with influenza, which impacted the workshop series and weekly meeting dynamics of the cohort. It is possible that this program organization challenge carried over into organization and correctness issues. Another hypothesis that we intend to test is potential differences between the three REU program cohorts in terms of composition (e.g., male/female ratio, primarily undergraduate institution vs. R1 institution, ratio of engineers to non-engineers).

Table 3. Concept Map Rubric Results for REU Cohorts

\begin{tabular}{|c|c|c|c|c|c|}
\hline Year & Dimension & $\begin{array}{c}\text { Pre-REU } \\
\text { Score }\end{array}$ & $\begin{array}{c}\text { Post-REU } \\
\text { Score }\end{array}$ & $\begin{array}{c}\text { p-value } \\
(\mathbf{t})\end{array}$ & $\begin{array}{c}\text { p-value } \\
\text { (Wilcoxon) }\end{array}$ \\
\hline \multirow{3}{*}{2012} & Comprehensiveness & 1.72 & 2.06 & .242 & .230 \\
\cline { 2 - 6 } & Organization & 2.00 & 2.06 & .880 & .943 \\
\cline { 2 - 6 } & Correctness & 2.44 & 2.56 & .594 & .516 \\
\cline { 2 - 6 } & Total & 6.17 & 6.67 & .376 & .461 \\
\hline \multirow{3}{*}{2013} & Comprehensiveness & 1.68 & 1.86 & .459 & .465 \\
\cline { 2 - 6 } & Organization & 2.27 & 1.96 & .132 & .131 \\
\cline { 2 - 6 } & Correctness & 2.36 & 2.05 & .089 & .083 \\
\cline { 2 - 6 } & Total & 6.32 & 5.86 & .257 & .177 \\
\cline { 2 - 6 } & Comprehensiveness & 1.65 & 1.85 & .168 & .157 \\
\cline { 2 - 6 } & Organization & 2.85 & 2.00 & .468 & .417 \\
\cline { 2 - 6 } & Correctness & 5.90 & 2.65 & .244 & .236 \\
\cline { 2 - 6 } & Total & 6.50 & .058 & .048 \\
\hline
\end{tabular}

In review of the findings from the other two cohorts, it was observed that 2012 had the highest total post score obtained at a mean value of 6.67, whereas 2014 had the highest difference in 
total scores between the two concept map measurements changing from 5.90 to 6.50 . The difference in the total concept map score in this year was seen to just reach the significant level at 0.048 based on the non-parametric test used for low sample size but was no longer significant after applying the Bonferroni adjustment for multiple comparisons. The change in individual student total scores for 2014 can be observed in Figure 2.

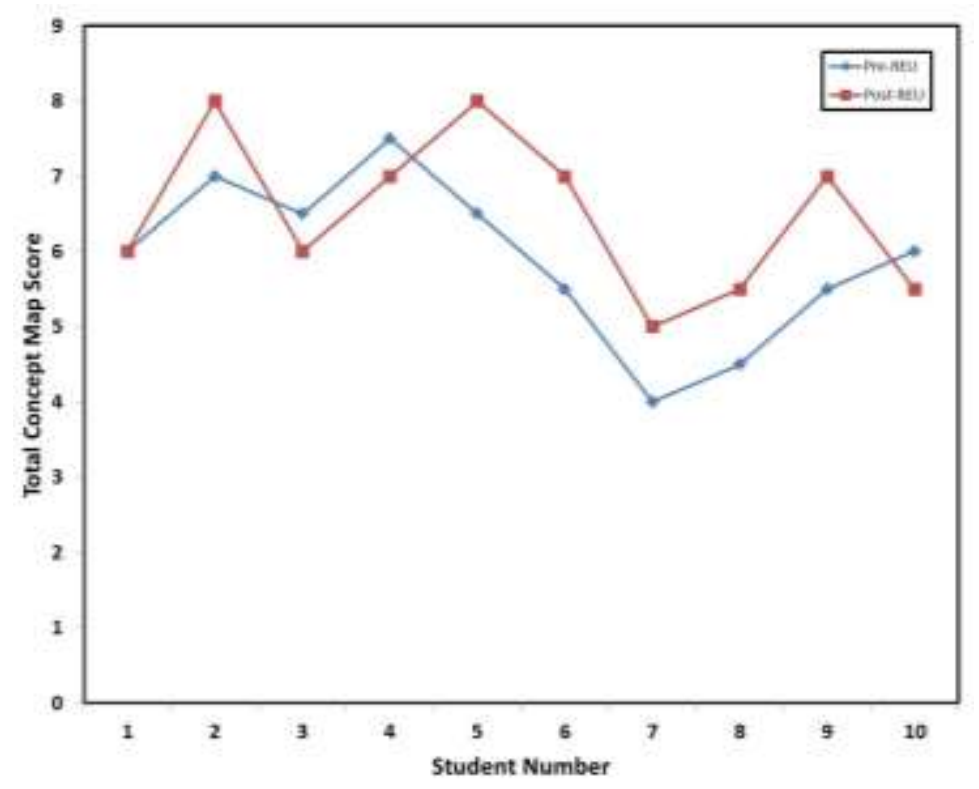

Figure 2. Student Total Concept Map Scores in 2014 REU Program

This figure shows how, with the exception of just three students within the ten student cohort, all remaining students showed an increase in their understanding/perception of the systems medicine field over the ten week REU program. In particular student five, whose maps are shown in Figures $1 \mathrm{a}$ and $\mathrm{b}$, demonstrated a 1.5 increase in total concept map score during the REU program. A similar increase was also observed in student six who participated as part of the same cohort. One of these two students was from a science department at a primarily undergraduate institution, where one would not expect significant exposure to Systems Medicine. As a result, this hard-working student was able to dramatically increase her knowledge base over the duration of the program. The other student was a post-sophomore biomedical engineering major from a research-I institution. Her project had her embedded in a clinical department and in close collaboration with a broad cross-section of PhD's and clinician MD's performing independent and clinically-sponsored research studies. It is likely that her map score improvement was driven by her expanded knowledge of the clinical, rather than academic side of systems medicine. Her overall program performance was outstanding, and she was among the five individuals invited to participate in the AIChE National Student Poster Competition as a result. A future analysis will look for specific linkages between the change in understanding/perception observed versus traits of the undergraduate students such as gender, ethnicity, undergraduate major and type of undergraduate institution.

Examination of student performance on the individual dimensions of the rubric for each cohort demonstrated that comprehensiveness was shown to slightly but not significantly increase in 
each year. This demonstrates that the REU program is capable of providing students with a solid understanding of key elements related to the systems medicine field. It also highlights areas, such as industrial applications and the interrelationships between academic disciplines and clinical practice that should be more explicitly addressed in the program workshop series during the summer program in order to achieve higher level comprehensiveness scores. Results obtained from the other two dimensions, organization and correctness, did not demonstrate any repeatable patterns between the different cohort years. The difference in these results may be due to the lack of emphasis on organization within the REU program where the main focus was on providing students with a clearer understanding of the field and the breadth it encompasses. Correctness may also vary based on an individual students' understanding and the particular mentor that they were assigned to work with. In this case, some students may have a very solid understanding of one particular area of systems medicine but they might still have uncertainty between how other areas of the field connect with one another.

One thing that cannot be determined from the scoring is students' experiences after leaving the program. One of the roles of a Research Experience for Undergraduates is to increase student desire to continue their studies, in the field if they so choose, at a post-graduate level or through a professional career. While a number of students have followed this path (including, as a noncomprehensive list, MS or PhD students within the field currently enrolled at RPI, Duke, Cornell, U. Rochester, and U. Pittsburgh), more recent cohorts are "too young" to have such data as they are still enrolled as undergraduates. It would be helpful to follow up with these individuals and the others that have participated in the program to gain a better understanding of how this REU experience shaped their future career decisions.

\section{Conclusions}

Systems medicine, or translational systems biology, is a highly diverse area of study that is still evolving. Engineers can benefit from gaining exposure to this field as it provides novel career opportunities and helps them develop an understanding for how diverse disciplines can work together to solve complex and challenging problems, such as cancer and other diseases. Research Experience for Undergraduates (REU) programs are, at present, the primary route for undergraduate students, particularly from primarily undergraduate institutions, to interact and engage with this field. The mentored research experience, along with professional development training over the course of a ten week period, provides an opportunity for students to explore challenging research problems with strong potential for societal impact.

Concept map scoring analysis demonstrated that the program at the University of Pittsburgh was capable of slightly but not significantly increasing students' comprehensiveness of the systems medicine field. It was also observed that the 2014 cohort of students showed the greatest change in total concept map score from the start to the end of the program. Experience of the program faculty, and the principal investigator, is likely the primary driver of the most recent cohort demonstrating some of the strongest gains as part of the program. Another potential effect is that the student applicant cohort for 2014 was markedly stronger than the 2013 or 2012 cohorts, as a result of the program "gaining a reputation" for positive student experiences and the development of strong relationships between the University of Pittsburgh and primarily undergraduate institutions such as Swarthmore, Smith, St. Lawrence, etc. In this way, students 
returning to their home institution can help the local faculty identify future students in their program likely to benefit from such an undergraduate research experience and actively promote them to the University of Pittsburgh REU program principal investigator. These "pipelines" offer the potential to enhance undergraduate research experiences for students from primarily undergraduate institutions by increasing confidence from the host REU program that students from schools with lasting relationships will be well-suited to a productive summer research experience.

Future analyses will examine potential correlations between the changes in student scores observed in Table 3 and the characteristics of the student, i.e. gender, ethnicity, degree program or undergraduate institution. It will also be of interest to evaluate the direct feedback from REU participant exit surveys as to how this program contributed to their further career progression as of the end of the program, as well as the opportunity to conduct follow-up surveys with students who have graduated from their undergraduate institution to evaluate the role the REU program has played in their current career selection.

\section{Acknowledgements}

This material is based upon work supported by the National Science Foundation (NSF EEC1156899). Any opinions, findings, and conclusions or recommendations expressed in this material are those of the author(s) and do not necessarily reflect the views of the National Science Foundation. Author Robert Parker also acknowledges financial support from the B.P. America Faculty Fellowship of the Swanson School of Engineering at the University of Pittsburgh. We also thank the Department of Chemical and Petroleum Engineering for staff support of this REU program.

\section{References}

1. Congressional Budget Office. Technological Change and the Rate of Health Care Spending. Pub. No. 2764. The Congress of the United States, 2008.

2. Besterfield-Sacre, M., Gerchak, J., Lyons, M., Shuman, L.J., Wolfe, H. (2004). Scoring Concept Maps: An Inegrated Rubric for Assessing Engineering Education. Journal of Engineering Education. 93(2), 105-115.

3. National Academy of Engineering Grand Challenges (2014). http://www.engineeringchallenges.org/cms/challenges.aspx. Accessed on January 21, 2015.

4. Coordinating Action Systems Medicine: Implementation of Systems Medicine across Europe. What is Systems Medicine? https://www.casym.eu/what-is-systems-medicine/ . Accessed on January 15, 2015.

5. Trefois, C., Antony, P. M. A., Goncalves, J., Skupin, A., Balling, R. (2015). Critical transitions in chronic disease: Transferring concepts from ecology to systems medicine. Current Opinion in Biotechnology, 34, 48-55.

6. Kim, N., He, N., Yoon, S. (2014). Cell line modeling for systems medicine in cancers (review). International Journal of Oncology, 44(2), 371-376.

7. Ho, T., Laird, C., Cao, Y., Parker, R.S. (2014). A Generalizable Optimization Algorithm for Clinically-Relevant Patient-Specific Cancer Chemotherapy Scheduling. Presented at the 2014 AIChE Annual Meeting, Atlanta, GA, November 2014, paper 282b.

8. Carcillo, C., Parker, R.S. (2014). A Biologically-Motivated Model of Chemotherapy-Induced Thrombocytopenia. Presented at the AIChE Annual Meeting, Atlanta, GA, November 2014, paper 200d. 
9. Ho, T., Parker, R.S. (2014). Personalizing Cancer Chemotherapy Treatment -- Coupling Clinical Data, Models, and Decision Support. EMBC 2014 - IEEE Engineering in Medicine and Biology Society Annual Meeting, Chicago, IL, August 2014.

10. Sheng, H., Landers, R. G., Liu, F., Nguyen, T. (2014). A longitudinal study on the effectiveness of the Research Experience for Undergraduates (REU) program at Missouri University of Science and Technology. Paper presented at the ASEE Annual Conference and Exposition, Conference Proceedings.

11. Norusis, M. (2005). SPSS 14.0 statistical procedures companion. Upper Saddle River, NJ: Prentice Hall, 438$439,442$.

12. Fleiss, J.L. (1986). The Design and Analysis of Clinical Experiments. New York: John Wiley \& Sons, 7. 